The value found will be in a decimalized form between $0-90^{\circ}$ and the true azimuth must be either $360 \pm Z$ or $180 \pm Z$ as common sense requires.

The sextant altitude is now corrected in accordance with normal practice and compared with the computed altitude. Depending on the conditions under which the sight was taken, limits are imposed on the intercept in judging the acceptability of the DR position. Given very good all-round conditions, a limit of $\angle O_{1}{ }^{\prime}$ is imposed and with poor conditions a limit of $\left\langle 0_{3}^{\prime}\right.$. These limits may sound tight but with experienced observers they have been found fairly generous on average.

There are several good reasons for using a pro-forma sheet instead of performing the whole computation by computer. The first is that seldom can the operation be performed without some interruption. Secondly the calculator has not enough storage facilities for all the required variables. Thirdly, the pro-forma sheet provides both a check on the progress of the computation and a check for eliminating mistakes.

The method described is particularly suited to Sun and Moon sights but multiple star sights have been found unduly cumbersome and are best plotted by the old and trusted method on a plotting sheet, allowing for runs.

\title{
Error Distribution in Navigation
}

\section{O. D. Anderson}

IN an earlier paper the present writer drew attention to the distinction between mixed and aggregated distributions. Incidentally, two minor mis-statements in that paper should be corrected. The first sentence in the last paragraph of section 2 (page 72 ) should read 'As $\sigma_{2} / \sigma_{1}$ ( $=k$, say) tends to either zero or infinity, $X / \sigma$ increases indefinitely; whilst for $k$ nearer unity, $X / \sigma$ is smaller'. Also the last line of the Appendix should not terminate with ' $X / \sigma=3.724$ ' but with ' $X / \sigma \rightarrow \infty$ '.

Mixed distributions arise when for example an error occurs either from one distribution with probability $p$, or from another with probability $1-p$, as in a set of position line errors due sometimes to one and sometimes to the other of a pair of observers of differing precision. An aggregate distribution occurs when the error consists of, say, two sub-errors, one from each of the two distributions; as when Captain Flint marks the chart and Long John subsequently reads it.

It has been suggested to the writer that the analysis for the mixed situation is all very well, but only the very special case of mixing equal numbers of observations from just two guassian distributions was considered. We here offer a rather more general theory which may be of wider interest, as for instance in quality control in a factory where a product might have been made on either a new or an older machine.

In many empirical situations it is found that observed distributions, though unimodal and symmetric, have sharper peaks and higher tails than the samevariance normal distribution. Indeed this has sometimes led to analysts playing safe and making a laplacian (two sided negative exponential) rather than a gaussian assumption. This non-normal behaviour can however be reconciled to a 
'normal' world, at least qualitatively, if we observe that these distributions are often evidently derived by mixing heterogeneous sets of data.

Consider the normal distributions:

$$
f_{i}(x)=\left(2 \pi \sigma_{i}^{2}\right)^{-1 / 2} \exp \left\{-x^{2} / 2 \sigma_{i}^{2}\right\} \quad i=\mathrm{I}, \ldots, n
$$

where, without loss in generality, $0<\sigma_{1} \leq \sigma_{2} \leq \cdots \leq \sigma_{n}$, though we exclude the homogeneous case where all the $\sigma_{i}$ are the same. Let $\left\{w_{i}: i=1, \ldots, n\right\}$ be a set of associated non-negative weights satisfying:

$$
\sum_{i=1}^{n} w_{i}=1 \text {. }
$$

The general mixed distribution is then defined by:

$$
f_{\mathrm{m}}(x)=\sum_{i=1}^{n} w_{i} f_{i}(x)
$$

and evidently has its variance given by:

where

$$
\sigma_{\mathrm{m}}^{2}=\sum_{i=1}^{n} w_{i} \sigma_{\mathrm{i}}^{2}
$$

$$
\sigma_{1}<\sigma_{\mathrm{m}}<\sigma_{\mathrm{n}} \text {. }
$$

Then using a result proved at the end of this paper, the same-variance gaussian distribution, $f_{\mathbf{8}}(x)$ say, obviously satisfies :

$$
f_{\mathrm{g}}(0)=\left(2 \pi \sum_{i=1}^{n} w_{i} \sigma_{i}^{2}\right)^{-1 / 2}<(2 \pi)^{-1 / 2} \sum_{i=1}^{n} w_{i} / \sigma_{i}=f_{\mathrm{m}}(0) .
$$

And, given large enough $X$, for all $x>X$, then $f_{\mathrm{g}}(x)<f_{\mathrm{m}}(x)$, since as $x \rightarrow \infty$, $\exp \left\{-x^{2} / 2 \sigma_{m}^{2}\right\} / \exp \left\{-x^{2} / 2 \sigma_{1}^{2}\right\} \rightarrow 0$.

Thus we have our qualitative explanation. It is also clear that $f_{\mathrm{g}}(x)$ and $f_{\mathrm{m}}(x)$ must cut each other an even number of times in the range $x>0$. Moreover, as for a gaussian distribution, $f_{\mathrm{m}}(x)$ has exactly one positive point of inflection.

Proof: A probability density function, $g_{i}(x)$, will be said to have the property $P$ if it has exactly one positive point of inflection, say at $x=G_{l}$, and $d^{2} g_{1}(x) / d x^{2}<0$ at $x=0$.

Consider any two general probability density functions having property $P$, say $g_{\mathrm{a}}(x)$ and $g_{\mathrm{b}}(x)$; where, without loss in generality, $G_{\mathrm{a}} \leq G_{\mathrm{b}}$. Choose any $\lambda$ between zero and unity. Then it immediately follows that $g_{\mathrm{j}}(x) \equiv \lambda g_{\mathrm{a}}(x)+$ $(1-\lambda) g_{b}(x)$ also has property $P$, with $G_{a} \leq G_{1} \leq G_{b}$.

Thus by induction, since all the $f_{i}(x)(i=1, \ldots, n)$ clearly have property $P$, so does $f_{m}(x)$, and its inflection is at a point strictly between $x=\sigma_{1}$ and $x=\sigma_{n}$. Of course the behaviour of $f_{m}(x)$, for $x<0$, follows from symmetry.

A special case arises when $n=2$ and $\sigma_{2}=k \sigma_{1},=k \sigma$ say.

$$
\begin{aligned}
f_{\mathrm{m}}(x) & =w_{1} f_{1}(x)+w_{2} f_{2}(x) \\
& =\frac{1}{(2 \pi)^{1 / 2} \sigma}\left[w_{1} \exp \left\{-x^{2} / 2 \sigma^{2}\right\}+\frac{w_{2}}{k} \exp \left\{-x^{2} / 2 k^{2} \sigma^{2}\right\}\right] \\
f_{\mathrm{g}}(x) & =\frac{1}{(2 \pi)^{1 / 2} \sigma\left(w_{1}+w_{2} k^{2}\right)^{1 / 2}} \exp \left\{-x^{2} / 2 \sigma^{2}\left(w_{1}+w_{2} k^{2}\right)\right\}
\end{aligned}
$$

so $f_{\mathrm{m}}(x)$ and $f_{\mathrm{g}}(x)$ cut in two points which are given by solving:

$w_{1} \exp \left\{-\frac{x^{2}}{2 \sigma^{2}}\left(I-\frac{I}{k^{2}}\right)\right\}+\frac{w_{2}}{k}=\frac{1}{\left(w_{1}+w_{2} k^{2}\right)^{1 / 2}} \exp \left\{-\frac{x^{2}}{2 \sigma^{2}}\left(\frac{I}{w_{1}+w_{2} k^{2}}-\frac{I}{k^{2}}\right)\right\}$. 
Putting

(2) reduces to

$$
z=\exp \left\{-\frac{x^{2} w_{1}\left(k^{2}-1\right)}{2 \sigma^{2}\left(w_{1}+w_{2} k^{2}\right) k^{2}}\right\}
$$

$$
w_{1} z^{\left[1+\left(w_{2} / w_{1}\right) k^{2}\right]}+\frac{w_{2}}{k}=\frac{\mathrm{I}}{\left(w_{1}+w_{2} k^{2}\right)^{1 / 2}} z
$$

which, for $w_{1}=w_{2}=\frac{1}{2}$, gives equation (4) of my previous paper. 1

For any required choice of $w_{1}, w_{2}$ and $k$, we can superimpose separate plots of the two sides of (3), as shown schematically in Fig. 1 . The points where the pair of plots cut give the (two) solutions of (3) and we are interested in the smaller of these, say $Z$, which corresponds to the larger solution of (2), say $X$.

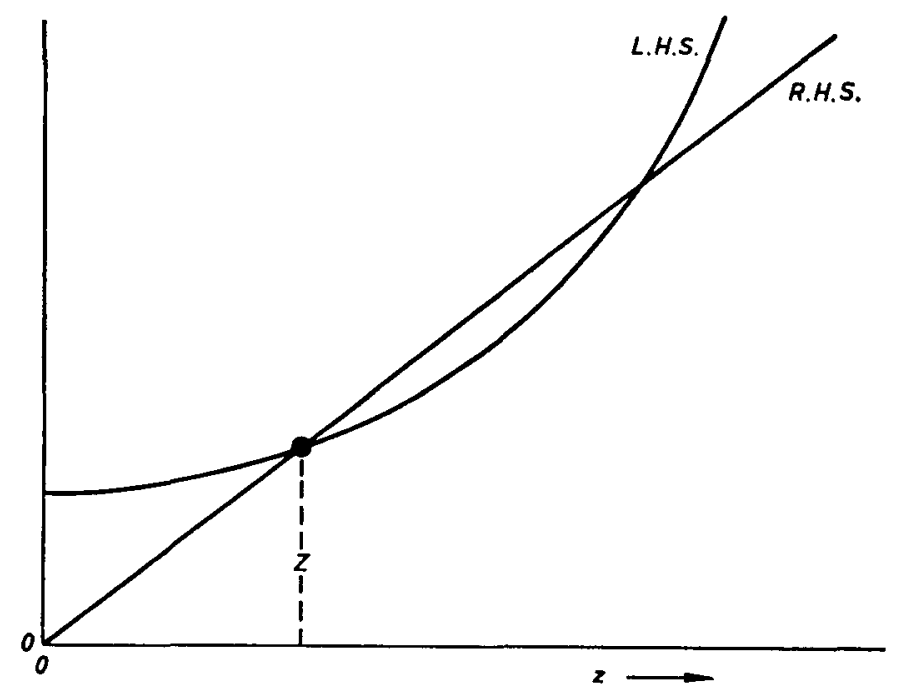

FIG. I. Schematic solution of equation (3)

Coincident solutions can only occur when $k=1$, and then $Z=1$ and $X$ is infinite. This is of course the trivial possibility of mixing identical distributions, which is the excluded homogeneous case.

The general situation of mixing any number $n$ of gaussians gives, corresponding to (3), an equation $H(z)=0$ where $H(z)$ is a sum of decreasing powers of $z$, with one negative coefficient and all the rest positive. Due to (I) the negative coefficient is neither the first nor the last, so $H(z)$ has exactly two changes of sign. Thus, from a version of Descartes' rule generalized to take care of the not necessarily integral powers, we have that the even number of solutions mentioned earlier must in fact be exactly two. The coincident case is again the trivial excluded one of $n$ identical gaussians. So the differences between the mixed distribution and the same-variance gaussian will always be as those shown schematically in Fig. 2.

The proof of the proposition :

$$
\sum_{i=1}^{n} \frac{w_{i}}{\sigma_{i}}>\left(\sum_{i=1}^{n} w_{i} \sigma_{i}^{2}\right)^{-1 / 2}
$$






FIG. 2. Schematic comparison between any general $f_{m}(x)$ and its corresponding $f_{\mathrm{g}}(x)$

is as follows. Since all the $w_{i}$ and $\sigma_{i}$ are positive it is sufficient to prove that:

$$
\sum_{i=1}^{n} w_{i} \sigma_{i}^{2}>\left(\sum_{i=1}^{n} \frac{w_{i}}{\sigma_{i}}\right)^{-2}
$$

Consider all the $w_{i}$ replaced by rational numbers $m_{i} / M$, where $\sum_{i=1}^{n} m_{i}=M$. (Even any irrationals can be approximated as closely as we like, for sufficiently large $M$ ).

Then, remembering that the $\sigma_{i}$ are not all equal, from Tchebychef's inequality:

$$
\sum_{i=1}^{n} w_{i} \sigma_{i}^{2}=\frac{1}{M} \sum_{i=1}^{n} m_{i} \sigma_{i}^{2}>\frac{1}{M^{2}}\left(\sum_{i=1}^{n} m_{i} \sigma_{i}\right)^{2} \text {. }
$$

So, since the arithmetic mean for unequal numbers is greater than the harmonic mean, as required:

$$
\sum_{i=1}^{n} w_{i} \sigma_{i}^{2}>\left[\frac{M}{\sum_{i=1}^{n}\left(m_{i} / \sigma_{i}\right)}\right]^{2}=\left(\sum_{i=1}^{n} \frac{w_{i}}{\sigma_{i}}\right)^{-2}
$$

\section{REFERENCE}

1 Anderson, O. D. (1976). On error distributions in navigation. This Journal, $29,69$.

\section{The Concept of Jocobrad}

Jocobrad (devised by K. D. Jones, G. R. Cowap and M. R. Bradshaw) has been devised in the light of research into the reactions and responses of mariners to various computer-based radar plotting and forecasting systems. It is a new concept in computer-based radar plotting systems which allows the 